\title{
Telomerase and the search for the end of cancer
}

Simone Mocellin ${ }^{1}$, MD, PhD, Karen A. Pooley ${ }^{2}$, MD, and Donato Nitti ${ }^{1}$, MD.

1 Department of Surgery, Oncology and Gastroenterology; School of Medicine, University of Padova, 35100 Padova, Italy

${ }^{2}$ Cancer Research UK Genetic Epidemiology Unit, Department of Public Health and Primary Care, Strangeways Research Laboratory, Cambridge,UK, e-mail: karen.pooley@srl.cam.ac.uk

Corresponding author: $\quad$ Simone Mocellin, $\mathrm{MD}, \mathrm{PhD}$

simone.mocellin@unipd.it (mocellins@hotmail.com)

Key words: telomerase, TERT, telomere, cancer, targeted therapy, immunotherapy, small molecule inhibitors, gene therapy

Conflict of interest: none declared 


\section{Summary}

Many of the fundamental molecular mechanisms underlying tumor biology remain elusive and, thus, developing specific anticancer therapies remains a challenge. The recently discovered relationships identified among telomeres, telomerase, aging, and cancer have opened a new avenue in tumor biology research that may revolutionize anticancer therapy. This review summarizes the critical aspects of telomerase biology that underpin the development of novel telomerase-targeting therapies for malignant diseases, and special regard is given to the aspects of telomerase that make it such an appealing target, such as the wide-spread expression of telomerase in cancers. Despite significant progress, issues remain to be addressed before telomerase-based therapies are truly effective and we include critical discussion of the results obtained thus far. 


\section{Telomeres and telomerase}

A classic cancer hallmark is the ability of malignant cells to proliferate indefinitely [1]. This is in stark contrast to the limited number of cell divisions normal somatic cells undergo (the so-called Hayflick limit) [2]. Today we know this restriction is due to the shortening of telomeres [3], the tandem repeat DNA sequence TTAGGG that associates with telomere binding proteins [4] to form the shelterin complex (Figure 1). This structure protects linear chromosome ends from recognition as DNA double-strand breaks (DSBs), thus contributing to genomic stability [5,6]. The fourstranded planar stacks that form within or between the guanine-rich single-strand of telomeric DNA (so-called G-quadruplexes) are a second conformation that protects the ends of DNA [7]. Due to the inability of the DNA replication machinery to copy the extreme ends of chromosomes, a phenomenon referred to as the "end replication problem", most human somatic cells show progressive telomere erosion as a consequence of ongoing cell division. Once a subset of telomeres reach a critically shortened length, a DNA damage response (DDR) is induced that triggers p53dependent G1/S cell cycle arrest, known as replicative senescence. Therefore, telomeres function not only as "caps" to protect chromosome ends from events such as fusion, degradation, or recombination but also serve as a biological clock to gauge (replicative) cell aging.

While looking for the solution to the "end replication problem", investigators discovered the holoenzyme telomerase [8], a ribonucleoprotein that synthesizes the hexameric tandem repeats onto the ends of eukaryotic chromosomes. The telomerase complex consists of two components, the reverse transcriptase catalytic subunit (TERT) and the telomerase RNA component (TERC). Telomerase helps maintain genome integrity as well as replication capability in both embryonic stem cells and proliferating progenitor cells derived from quiescent normal stem cells (e.g., male germ-line spermatocytes), but it is silent in somatic cells, which make up the vast majority of human tissues [9].

\section{Telomerase and cancer}

In the early 1990s, the discovery of a relationship between telomeres, telomerase, aging, and cancer $[10,11]$ opened an entirely new avenue in tumor biology research, with potentially revolutionary implications for anticancer therapy [12,13]. Unlike most normal human cells - which lack telomerase activity and experience telomere shortening with each cell division until they enter replicative senescence - cells deficient in cell cycle checkpoints (e.g., early stage malignant cells 
that lack p53, p16, or ARF/CDKN2A) escape replicative senescence and continue to divide (Figure 1). These cells eventually enter a second growth arrest state (crisis) when many shortened chromosome ends fuse, leading to cycles of chromosome bridge-breakage-fusion that almost universally result in apoptosis. In human cells, these two mechanisms of restricting cell growth (senescence and crisis) are potent mechanisms for protecting against cancer. Most human cells remain in this crisis period, with cell growth being balanced by cell death, but on rare occasions a cell acquires a mechanism, such as telomerase expression, that can maintain or lengthen telomeres. Cells that have escaped crisis are usually characterized by two hallmarks: telomere stability and the reactivation of telomerase expression. This rare type of cell can then grow continuously (i.e., it becomes immortal), and this is believed to be a pivotal step in carcinogenesis. Indeed, in the presence of an intact p53 pathway, mice that lack telomerase activity are resistant to cancer development [14-16], whereas forced TERT overexpression in different mouse models is associated with an increased incidence of spontaneous tumors [17,18].

In addition to its canonical role in maintaining telomere length in malignant cells, telomerase has recently been recognized to interfere with extra-telomeric tumor-promoting pathways [9]. For instance, TERT can function as a transcriptional modulator of $\mathrm{Wnt} /$ beta-catenin signaling by serving in combination with BRG1 (a SWI/SNF-related chromatin remodeling protein) as part of a beta-catenin transcriptional complex. Additional evidence also supports the role of telomerase in the regulation of apoptosis in a non-canonical, telomere-independent manner. The overexpression of TERT - which contains a mitochondrial localization signal peptide - suppresses programmed cell death, and TERT downregulation enhances the mitochondrial apoptotic pathway by the post-translational activation of the proapoptotic factor Bax. In light of the pivotal importance of both the Wnt/beta-catenin pathway and apoptosis in cancer biology, these experimental findings strengthen the hypothesis that telomerase has a central role in cancer development and progression.

Ex vivo studies carried out with human tissues have shown telomerase to be expressed in approximately $90 \%$ of all malignant tumors [19,20], which makes it a potential biomarker for most cancers. Furthermore, telomerase levels correlate with the severity of patient prognosis for a variety of tumor types, and so it may become a predictor of clinical outcome in different settings [21,22]. Finally, epidemiological studies on the relationship between cancer incidence and polymorphisms in the 5p15.33 locus, which encodes TERT, strongly suggest that variability of the TERT gene sequence affects individual cancer predisposition [23], although the molecular mechanisms underlying this association are still unclear. 


\section{Telomerase targeting anticancer strategies}

Considering the evidence mentioned above linking telomerase activity to tumor development and progression, researchers are devising a variety of methods to target telomerase as a novel therapy against cancer [12,13] (Figure 2). As for any targeted approach, telomerase-based antitumor strategies hinge upon specificity for the target, which allows the treatment to affect malignant cells with little, or ideally no, toxicity for healthy tissues. Telomerase is activated in the majority of human tumors $[13,19]$, and the 5 p15.33 locus is frequently amplified in many types of cancer [24], which provides malignant cells with the capacity to divide indefinitely. Overall, these observations suggest that targeted inhibition of telomerase could trigger critically short telomeres and, ultimately, lead to a selective loss of cell viability in the tumor. However, telomerase expression is also observed in a variety of normal tissues (e.g., bone marrow blood cells, basal layer skin cells, and epithelial cells of mucosae), at least during the S-phase of the cell cycle when telomerase activity is mainly growth-regulated [25-28]. In fact, actively dividing cells maintain strictly regulated telomerase expression and activity, whereas telomerase expression is repressed in differentiated somatic tissues. By contrast, most tumor cells show a high level of telomerase activity, and tumor cells - upon oncogenic stimuli - accelerate their proliferation rate, consequently decreasing the length of their telomeres and explaining why telomeres are usually shorter in malignant cells compared with surrounding healthy tissues [13,29-32]. As a consequence, normal progenitor cells and stem cells, having relatively long telomeres and undergoing less frequent mitosis, should be more resilient to the therapeutic inhibition of telomerase compared with malignant cells. This consideration, along with telomerase overexpression in malignant versus healthy tissues, is the fundamental rationale for the telomerase-targeted anticancer strategies discussed below.

\section{$\underline{\text { Small molecule inhibitors }}$}

The most intuitive approach for therapeutically exploiting the role of telomerase in cancer biology is to inhibit its enzymatic activity. This can be achieved using small synthetic molecules such as BIBR1532 (2-[[E]-3-naphthalene-2-yl-but-2-enoylamino]-benzoic acid), a noncompetitive inhibitor of both TERT and TERC [33]. In a range of human cancer cell lines derived from both solid tumors and hematological malignancies, in vitro treatment with this drug reduces telomere length, inhibits cell proliferation, leads to cellular senescence, and - at higher doses - is cytotoxic [34,35]. When cancer cells pretreated with BIBR1532 are implanted into nude mice, tumor growth is significantly 
delayed compared with a control group, although additional treatment does not produce any further advantage [34]. Interestingly, the cytotoxic effect appears to be selective for malignant cells of the hematopoietic system because the proliferative capacity of normal CD34(+) cells from cord blood and leukapheresis samples is not affected by treatment with BIBR1532 [35].

An indirect strategy for inhibiting telomerase activity is stabilization of G-quadruplexes, which prevents TERC from recognizing the hydroxyl group at the 3' end of the unfolded, single-stranded telomere overhang [7]. So-called G-quadruplex ligands are small synthetic molecules that stabilize telomere quadruplex structures, thus inhibiting telomerase activity [36-38]. The macrocyclic compound telomestatin, derived from Streptomyces anulatus, is the best characterized of these ligands and potently inhibits telomerase [39]. This drug, as well as others such as the tri-substituted acridine compound BRACO19, have promising anticancer effects both in vitro and in vivo in preclinical models [40-42], including the eradication of cancer stem cells by the induction of apoptosis [43].

The clinical testing of small molecule telomerase inhibitors is hampered by poor pharmacokinetic characteristics, such as limited solubility in water, which requires the addition of solubilization enhancers or drug carrier systems to make these compounds bioavailable. Although BRACO19 is more soluble than other small molecule inhibitors, its use is still limited by the low permeability that renders it unable to pass through biological barriers in airways or intestinal epithelial cell cultures in vitro [44]. This key issue is underscored by an elegant in vivo experiment demonstrating that oral administration of BRACO19 has no therapeutic effect, whereas intraperitoneal injection leads to significant tumor shrinkage [42]. More hydrophobic compounds have been designed to circumvent this issue, including RHPS4 [45] and quarfloxin/CX-3543; quarfloxin has been evaluated in a phase II clinical trial as a treatment for neuroendocrine tumors [46], although results are not yet available.

\section{Immunotherapy}

Active immunization (i.e., vaccination) against tumor associated antigens (TAAs) has been long proposed as a "smart" therapeutic approach against cancer, although the results in clinical settings have so far been disappointing [47,48]. Whereas most TAAs are specific for a tumor type (e.g., carcino-embryonic antigen [CEA] for gastrointestinal carcinomas or tyrosinase for melanoma), telomerase-based immunotherapy may be suitable for a variety of malignancies due to the almost ubiquitous expression of telomerase in malignant cells [49]. TERT-derived peptides can be 
recognized by cytotoxic CD8+ lymphocytes in a MHC class I restricted fashion [50], and TERTbased vaccination has been shown to lead to tumor regression in different preclinical models [5153] (reviewed in [49]).

In the clinical setting, most patients affected with various solid tumors and vaccinated with telomerase peptides mount an immunological response, as assessed by the frequency of epitope specific CD8+ T-lymphocytes in the peripheral blood, with no high grade toxicity being observed. This response has been seen for both unmodified peptides, such as GV1001, a 16-mer TERT peptide that binds multiple HLA class II molecules and harbors putative HLA class I epitopes [54] as well as optimized telomerase peptides, including Vx-001, a 9-mer HLA-A*0201 restricted TERT cryptic peptide modified at position 572 to increase its immunogenicity [55]. Similar data have been described for patients with prostate cancer vaccinated with GRNVAC1, a preparation of mature autologous dendritic cells transfected with a TERT-LAMP (lysosomal associated membrane protein) fusion construct, with the LAMP domain facilitating lysosome targeting and processing of the TAA TERT [56]. Importantly, a complete tumor response has been recorded in a patient with pancreatic carcinoma vaccinated with dendritic cells transfected with TERT mRNA [57], and patients with an immunological response to TERT peptides (e.g., I540 [58] or Vx-001 [59]) have been shown to survive longer than non-responders in non-randomized studies [58,59]. Although a lack of both immune and tumor response has also been reported in patients with hepatocellular carcinoma and cutaneous T-cell lymphoma treated with GV1001 [60,61], ongoing trials are formally testing the hypothesis that telomerase can be an effective target for anticancer immunotherapy in humans (Table 1).

\section{Gene therapy}

Despite the safety issues and the relatively recent implementation in the clinical setting, the gene therapy approach to human diseases has found its niche [62], although in the oncology field no such treatment has yet become the standard therapy for any tumor type [63-65]. Gene therapies involving TERT or TERC can be classified into two main types: (i) the inhibition of TERT or TERC expression/activity targeting their RNAs; or (ii) the utilization of TERT or TERC promoters to drive expression of exogenous vectors. In the first case, antisense oligonucleotides (ASOs, singlestranded DNA or RNA sequences complementary to the target RNA), small interfering RNAs (siRNAs, double-stranded RNA molecules that regulate both mRNA degradation and mRNA translation by the RNA interference phenomenon), and ribozymes (RNA enzymes capable of 
cleaving specific mRNAs) have been employed to specifically inhibit the activity or reduce the levels of TERT or TERC, which ultimately lowers the enzymatic activity of telomerase. In a variety of preclinical models this approach not only leads to senescence and apoptosis of human cancer cells in vitro [66-69] but also to significant antitumor effects in vivo [66-68,70].

Probably the most well-studied ASO in this context is imetelstat (also known as GRN163L), a lipidmodified 13-mer oligonucleotide N3'->P5'-thio-phosphoramidate complementary to the template region of TERC [71]. After showing therapeutic potential in preclinical models [66,70], including an ability to target cancer stem cells [72,73], this telomerase inhibitor is currently being tested in clinical trials (see Table 1).

The second telomerase-based gene therapy approach exploits the TERT promoter to deliver "suicide" genes or oncolytic viruses selectively to malignant cells: as for telomerase-based immunotherapy, this strategy does not aim to inhibit telomerase activity but rather exploit telomerase overexpression in tumors. For instance, a viral vector genetically modified to encode a prodrug activating enzyme, such as carboxypeptidase G2 or cytosine deaminase, and to replicate only in TERT-overexpressing cells can be used to activate the effect of the cytotoxic prodrug, such as ZD2767P or 5-flucytosine, respectively, only in the tumor [74,75].

Similarly, oncolytic viruses can be designed to induce virus-mediated lysis of tumor cells after selective viral propagation within the TERT-overexpressing tumor [76,77]. The latter strategy has already entered the clinical phase of experimentation with an attenuated adenovirus-5 vector (telomelysin/OBP-301) [78] in which the TERT promoter element drives the expression of E1A and E1B genes linked with an internal ribosome entry site (IRES). Telomelysin replicates efficiently and induces significant cell killing in a panel of human cancer cell lines, whereas replication and cytotoxicity are highly attenuated in normal human cells lacking telomerase activity [79].

\section{Challenges and future perspectives}

Telomerase research has opened a promising avenue in the fight against cancer, and preclinical findings provide new hope for patients affected with malignant diseases. However, the clinical implementation of telomerase-based therapeutic strategies is proceeding at a pace slower than was probably expected [80]. Among the three randomized controlled phase III trials of therapies targeting telomerase, one was prematurely stopped based on a lack of survival benefit for patients undergoing vaccination with GV1001 followed by administration of gemcitabine, as compared with patients receiving chemotherapy alone (trial NCT00358566 at 
www.clinicaltrials.gov), and two testing the efficacy of the GV1001 vaccine given after chemotherapy are ongoing (Table 1). The results of these latter studies are eagerly awaited to define the potential role of this therapeutic approach in prolonging patient survival. This apparent discrepancy between experimental and clinical data does not likely depend only on the intrinsic difficulty of testing novel cancer treatments in a clinical setting, but may also derive from many open questions regarding telomerase biology and targeting that need to be answered before the therapeutic potential can be fully exploited.

\section{The shortest telomere issue}

One of several open questions in the development of telomerase-targeting therapies remains 'what is the precise anticancer mechanism of telomerase inhibitors?' Telomere shortening, which ultimately causes apoptosis of malignant cells, is generally believed to be the most relevant mechanism as it can be observed as a result of a variety of telomerase inhibition methods [66,67,70]. Nevertheless, in some models this rule does not apply $[69,81,82]$, not only casting doubts on the way telomerase inhibitors act against cancer but also representing a serious hurdle in the search for predictors of tumor response. In fact, if the average telomere length consistently decreased upon administration of such drugs, its measurement might be proposed as a potentially useful biomarker for the early detection of tumor response (and thus drug effectiveness). However, since the shortest telomeres in a cell are those responsible for triggering a DNA damage checkpoint and/or genomic instability following telomerase inhibition [83], for the purposes of prediction it is much more informative to know the distribution of shortest telomeres in a tumor cell population (on a cell-by-cell basis) rather than to measure the average telomere length. Accordingly, only the development of reliable quantitative assays to measure the cell-based distribution of the shortest telomeres in a given cell population will allow oncologists to optimize the selection of cancer patients with the greatest likelihood of responding to telomerase inhibitors.

Finally, because short telomeres drive genomic instability and facilitate clonal evolution [9], there is the theoretical possibility that telomerase inhibition might exacerbate this process, which ultimately might accelerate tumor progression. Therefore, investigators should take into consideration this potential caveat while dealing with telomerase inhibition as a novel anticancer therapy.

\section{The perfect target}

There also appears to be conflicting evidence regarding the most suitable telomerase component for targeting (i.e., TERT versus TERC). In a comparative study, ASO-mediated inhibition of TERT 
induces growth arrest and apoptosis of human prostate cancer cells, whereas inhibition of the telomerase RNA component has no such effect [81]. Conversely, other investigators reported similar biological effects for both TERT and TERC inhibition, and the simultaneous inhibition of both components provided an additive effect [84]. Even more puzzling is the demonstration that in other experimental settings, for example when using the small molecule inhibitor BIBR1532 against leukemic cells, the enzyme activity of telomerase does not appear to be involved in the therapeutic effect of telomerase inhibition [35]. Clearly, only by defining the molecular events that lead to cancer cell death following telomerase targeting will enable investigators to make the most of antitelomerase therapies in the fight against cancer.

\section{$\underline{\text { Pharmacokinetic issues }}$}

Another key issue in the development of telomerase-based antitumor strategies is the pharmacokinetic properties of molecules targeting telomerase. As mentioned above, small molecules inhibitors are often insufficiently hydrophobic and thus scarcely penetrate through cell membranes, strongly limiting their use in vivo. Antisense oligonucleotides, siRNAs, and ribozymes are even bigger molecules that often have even worse diffusion characteristics and require association with lipid carriers such as oligofectamine or lipofectamine to increase their penetration into malignant cells. However, the lipid carriers are associated with non-negligible toxic effects on healthy cells, and therefore other solutions are being assessed for human trials. For instance, lipid modification (i.e., conjugation with a palmitoyl moiety) significantly augments the bioavailability of imetelstat/GRN163L, an ASO resistant to nuclease activity due to thio-phosphoroamidate modification of its backbone [71] that is currently being tested in clinical trials (Table 1). Similarly, nanotechnologies are providing researchers with novel tools to enhance the bioavailability of telomerase-targeting drugs. For example, delivery of anti-TERT siRNA using N-(6-aminohexyl)carbamate-modified single-walled carbon nanotubes suppresses the growth of human tumor xenografts [68]. Nevertheless, the clinical use of this carrier system is probably limited by the lack of biodegradability and unclear toxicology of carbon nanotubes; this inconvenience might be overcome by biodegradable polyethylenimine(PEI)-based nanoparticles, as recently demonstrated for siRNA molecules targeting TERT that inhibit tumor growth in a xenograft model [85].

\section{Combinatorial regimens}

As reported above, targeting telomerase with a variety of approaches can delay in vivo cancer growth in preclinical models. However, more pronounced anticancer effects such as shrinkage and possibly disappearance of already established tumor masses are desirable in the clinical setting. 
Moreover, the lag phase between when the telomeres have shortened to their critical limit and the onset of cell senescence or apoptosis may take several weeks. To tackle these hurdles, the combination of telomerase-based treatments with established anticancer therapies appears to be a promising approach; in fact, preclinical evidence supports the idea of synergistic anticancer effects for all of the above mentioned telomerase targeting strategies with regimens already approved for human cancer treatment, including chemotherapeutic agents (e.g., etoposide [86], docetaxel [87], doxorubixin [88], paclitaxel [89]), monoclonal antibodies (e.g., anti-HER2 trastuzumab [90]), immunotherapy (e.g., anti-CTLA4 antibodies [91]), and radiotherapy [92]. Considering the potential benefit of combinatorial approaches, both telomerase inhibitors and telomerase-based vaccines are being tested as maintenance/consolidation regimens to prolong remission after standard chemotherapy in patients with advanced cancers (see Table 1).

\section{$\underline{\text { Safety issues }}$}

Finally, safety concerns must also be considered. One issue not specifically linked to the target (telomerase) but to the vectors is the oncogenic potential of gene therapy approaches that hinge upon cell transfection with constructs encoded by viruses or plasmids [62,93]. Although the risk of causing secondary neoplasms may be considered of minor relevance when the prognosis is poor (i.e., in case of metastasis), this issue becomes crucial in patients with early stage cancer. In addition, the specific side effects following the inhibition of telomerase activity, or its expression, in humans remain to be fully evaluated. As reviewed here, the differences in telomerase expression and telomere length in healthy versus malignant tissues may make telomerase the safest cancer target identified to date. Nevertheless, telomerase is important for the renewal capacity of normal stem and progenitor cells [94]. Moreover, telomere length in the bone marrow shortens with age and can be further eroded by anticancer chemotherapy [95]; therefore, a long-term adverse effect of telomerase-based cancer therapies on normal tissues cannot be excluded a priori. Thus far no evidence of toxicity of this type has been reported in any of the proliferative tissues (including bone marrow) analyzed in animal models or clinical trials. However, experience is still relatively limited, and thus exploratory biomarkers of telomere status in proliferative tissues are under evaluation and ongoing trials will need to monitor such potential effects on multiple normal tissues.

Overall, the preclinical evidence strongly supports the therapeutic potential of telomerase based antitumor strategies and justifies further efforts for their clinical implementation. However, only by addressing the main issues mentioned above will investigators be able to assess whether the promise 
of telomerase targeting treatments can be translated into real benefit for patients afflicted with cancer. 


\section{References}

1 Hanahan, D. and Weinberg, R.A. (2011) Hallmarks of cancer: the next generation. Cell 144, 646-674

2 Hayflick, L. and Moorhead, P.S. (1961) The serial cultivation of human diploid cell strains. Exp Cell Res 25, 585-621

3 Bodnar, A.G. et al. (1998) Extension of life-span by introduction of telomerase into normal human cells. Science 279, 349-352

4 Moyzis, R.K. et al. (1988) A highly conserved repetitive DNA sequence, (TTAGGG)n, present at the telomeres of human chromosomes. Proc Natl Acad Sci U S A 85, 6622-6626

5 Blackburn, E.H. (2001) Switching and signaling at the telomere. Cell 106, 661-673

6 de Lange, T. (2009) How telomeres solve the end-protection problem. Science 326, 948-952

7 Gilson, E. and Geli, V. (2007) How telomeres are replicated. Nat Rev Mol Cell Biol 8, 825838

8 Greider, C.W. and Blackburn, E.H. (1989) A telomeric sequence in the RNA of Tetrahymena telomerase required for telomere repeat synthesis. Nature 337, 331-337

9 Martinez, P. and Blasco, M.A. (2011) Telomeric and extra-telomeric roles for telomerase and the telomere-binding proteins. Nat Rev Cancer 11, 161-176

10 Calado, R.T. and Young, N.S. (2009) Telomere diseases. N Engl J Med 361, 2353-2365

11 Artandi, S.E. (2002) Telomere shortening and cell fates in mouse models of neoplasia. Trends Mol Med 8, 44-47

12 Harley, C.B. (2008) Telomerase and cancer therapeutics. Nat Rev Cancer 8, 167-179

13 Shay, J.W. and Wright, W.E. (2006) Telomerase therapeutics for cancer: challenges and new directions. Nat Rev Drug Discov 5, 577-584

14 Blasco, M.A. et al. (1997) Telomere shortening and tumor formation by mouse cells lacking telomerase RNA. Cell 91, 25-34

15 Feldser, D.M. and Greider, C.W. (2007) Short telomeres limit tumor progression in vivo by inducing senescence. Cancer Cell 11, 461-469

16 Gonzalez-Suarez, E. et al. (2000) Telomerase-deficient mice with short telomeres are resistant to skin tumorigenesis. Nat Genet 26, 114-117

17 Gonzalez-Suarez, E. et al. (2001) Increased epidermal tumors and increased skin wound healing in transgenic mice overexpressing the catalytic subunit of telomerase, mTERT, in basal keratinocytes. Embo J 20, 2619-2630 
18 Artandi, S.E. et al. (2002) Constitutive telomerase expression promotes mammary carcinomas in aging mice. Proc Natl Acad Sci U S A 99, 8191-8196

19 Kim, N.W. et al. (1994) Specific association of human telomerase activity with immortal cells and cancer. Science 266, 2011-2015

20 Blasco, M.A. and Hahn, W.C. (2003) Evolving views of telomerase and cancer. Trends Cell Biol 13, 289-294

21 Langford, L.A. et al. (1997) Telomerase activity in ordinary meningiomas predicts poor outcome. Hum Pathol 28, 416-420

22 Clark, G.M. et al. (1997) Telomerase activity and survival of patients with node-positive breast cancer. J Natl Cancer Inst 89, 1874-1881

23 Mocellin, S. et al. (2012) Telomerase reverse transcriptase locus polymorphisms and cancer risk: a field synopsis and meta-analysis. J Natl Cancer Inst 104, 840-854

24 Hahn, W.C. and Meyerson, M. (2001) Telomerase activation, cellular immortalization and cancer. Ann Med 33, 123-129

25 Trujillo, K.A. et al. (2011) Breast field cancerization: isolation and comparison of telomerase-expressing cells in tumor and tumor adjacent, histologically normal breast tissue. Mol Cancer Res 9, 1209-1221

26 Shay, J.W. and Wright, W.E. (2010) Telomeres and telomerase in normal and cancer stem cells. FEBS Lett 584, 3819-3825

27 Kyo, S. and Inoue, M. (2002) Complex regulatory mechanisms of telomerase activity in normal and cancer cells: how can we apply them for cancer therapy? Oncogene 21, 688-697

28 Greider, C.W. (1998) Telomerase activity, cell proliferation, and cancer. Proc Natl Acad Sci U S A 95, 90-92

29 Gertler, R. et al. (2004) Telomere length and human telomerase reverse transcriptase expression as markers for progression and prognosis of colorectal carcinoma. J Clin Oncol 22, $1807-1814$

30 Kobitsu, K. et al. (1997) Shortened telomere length and increased telomerase activity in hamster pancreatic duct adenocarcinomas and cell lines. Mol Carcinog 18, 153-159

31 Raynaud, C.M. et al. (2010) DNA damage repair and telomere length in normal breast, preneoplastic lesions, and invasive cancer. Am J Clin Oncol 33, 341-345

32 Engelhardt, M. et al. (1997) Relative contribution of normal and neoplastic cells determines telomerase activity and telomere length in primary cancers of the prostate, colon, and sarcoma. Clin Cancer Res 3, 1849-1857 
33 Pascolo, E. et al. (2002) Mechanism of human telomerase inhibition by BIBR1532, a synthetic, non-nucleosidic drug candidate. J Biol Chem 277, 15566-15572

34 Damm, K. et al. (2001) A highly selective telomerase inhibitor limiting human cancer cell proliferation. Embo J 20, 6958-6968

35 El-Daly, H. et al. (2005) Selective cytotoxicity and telomere damage in leukemia cells using the telomerase inhibitor BIBR1532. Blood 105, 1742-1749

36 Li, Q. et al. (2012) Searching drug-like anti-cancer compound(s) based on G-quadruplex ligands. Curr Pharm Des 18, 1973-1983

37 Neidle, S. (2010) Human telomeric G-quadruplex: the current status of telomeric Gquadruplexes as therapeutic targets in human cancer. Febs $J$ 277, 1118-1125

38 De Cian, A. et al. (2007) Reevaluation of telomerase inhibition by quadruplex ligands and their mechanisms of action. Proc Natl Acad Sci U S A 104, 17347-17352

39 Kim, M.Y. et al. (2002) Telomestatin, a potent telomerase inhibitor that interacts quite specifically with the human telomeric intramolecular g-quadruplex. J Am Chem Soc 124, 2098-2099

40 Gomez, D. et al. (2006) The G-quadruplex ligand telomestatin inhibits POT1 binding to telomeric sequences in vitro and induces GFP-POT1 dissociation from telomeres in human cells. Cancer Res 66, 6908-6912

41 Tauchi, T. et al. (2003) Activity of a novel G-quadruplex-interactive telomerase inhibitor, telomestatin (SOT-095), against human leukemia cells: involvement of ATM-dependent DNA damage response pathways. Oncogene 22, 5338-5347

42 Burger, A.M. et al. (2005) The G-quadruplex-interactive molecule BRACO-19 inhibits tumor growth, consistent with telomere targeting and interference with telomerase function. Cancer Res 65, 1489-1496

43 Miyazaki, T. et al. (2012) Telomestatin impairs glioma stem cell survival and growth through the disruption of telomeric G-quadruplex and inhibition of the proto-oncogene, cMyb. Clin Cancer Res 18, 1268-1280

44 Taetz, S. et al. (2006) Biopharmaceutical characterization of the telomerase inhibitor BRACO19. Pharm Res 23, 1031-1037

45 Phatak, P. et al. (2007) Telomere uncapping by the G-quadruplex ligand RHPS4 inhibits clonogenic tumour cell growth in vitro and in vivo consistent with a cancer stem cell targeting mechanism. Br J Cancer 96, 1223-1233

46 Drygin, D. et al. (2009) Anticancer activity of CX-3543: a direct inhibitor of rRNA biogenesis. Cancer Res 69, 7653-7661 
47 Mocellin, S. et al. (2004) Part I: Vaccines for solid tumours. Lancet Oncol 5, 681-689

48 Klebanoff, C.A. et al. (2011) Therapeutic cancer vaccines: are we there yet? Immunol Rev $239,27-44$

49 Liu, J.P. et al. (2010) Telomerase in cancer immunotherapy. Biochim Biophys Acta 1805, $35-42$

50 Vonderheide, R.H. et al. (1999) The telomerase catalytic subunit is a widely expressed tumor-associated antigen recognized by cytotoxic T lymphocytes. Immunity 10, 673-679

51 Schmidt, J. et al. (2006) Telomerase-specific T-cells kill pancreatic tumor cells in vitro and in vivo. Cancer 106, 759-764

52 Nair, S.K. et al. (2000) Induction of cytotoxic T cell responses and tumor immunity against unrelated tumors using telomerase reverse transcriptase RNA transfected dendritic cells. Nat Med 6, 1011-1017

53 Peruzzi, D. et al. (2010) A vaccine targeting telomerase enhances survival of dogs affected by B-cell lymphoma. Mol Ther 18, 1559-1567

54 Brunsvig, P.F. et al. (2011) Telomerase peptide vaccination in NSCLC: a phase II trial in stage III patients vaccinated after chemoradiotherapy and an 8-year update on a phase I/II trial. Clin Cancer Res 17, 6847-6857

55 Mavroudis, D. et al. (2006) A phase I study of the optimized cryptic peptide TERT(572y) in patients with advanced malignancies. Oncology 70, 306-314

$56 \mathrm{Su}, \mathrm{Z}$. et al. (2005) Telomerase mRNA-transfected dendritic cells stimulate antigen-specific CD8+ and CD4+ $\mathrm{T}$ cell responses in patients with metastatic prostate cancer. J Immunol $174,3798-3807$

57 Suso, E.M. et al. (2011) hTERT mRNA dendritic cell vaccination: complete response in a pancreatic cancer patient associated with response against several hTERT epitopes. Cancer Immunol Immunother 60, 809-818

58 Domchek, S.M. et al. (2007) Telomerase-specific T-cell immunity in breast cancer: effect of vaccination on tumor immunosurveillance. Cancer Res 67, 10546-10555

59 Vetsika, E.K. et al. (2012) Immunological responses in cancer patients after vaccination with the therapeutic telomerase-specific vaccine Vx-001. Cancer Immunol Immunother 61, 157-168

60 Greten, T.F. et al. (2010) A phase II open label trial evaluating safety and efficacy of a telomerase peptide vaccination in patients with advanced hepatocellular carcinoma. BMC Cancer 10, 209 
61 Schlapbach, C. et al. (2011) Telomerase-specific GV1001 peptide vaccination fails to induce objective tumor response in patients with cutaneous T cell lymphoma. J Dermatol Sci 62, $75-83$

62 Sheridan, C. (2011) Gene therapy finds its niche. Nat Biotechnol 29, 121-128

63 Ortiz, R. et al. (2012) New gene therapy strategies for cancer treatment: a review of recent patents. Recent Pat Anticancer Drug Discov 7, 297-312

64 Duarte, S. et al. (2012) Suicide gene therapy in cancer: where do we stand now? Cancer Lett $324,160-170$

65 Petrocca, F. and Lieberman, J. (2011) Promise and challenge of RNA interference-based therapy for cancer. J Clin Oncol 29, 747-754

66 Dikmen, Z.G. et al. (2005) In vivo inhibition of lung cancer by GRN163L: a novel human telomerase inhibitor. Cancer Res 65, 7866-7873

67 Shammas, M.A. et al. (2008) Telomerase inhibitor GRN163L inhibits myeloma cell growth in vitro and in vivo. Leukemia 22, 1410-1418

68 Zhang, Z. et al. (2006) Delivery of telomerase reverse transcriptase small interfering RNA in complex with positively charged single-walled carbon nanotubes suppresses tumor growth. Clin Cancer Res 12, 4933-4939

69 Saretzki, G. et al. (2001) Ribozyme-mediated telomerase inhibition induces immediate cell loss but not telomere shortening in ovarian cancer cells. Cancer Gene Ther 8, 827-834

70 Hochreiter, A.E. et al. (2006) Telomerase template antagonist GRN163L disrupts telomere maintenance, tumor growth, and metastasis of breast cancer. Clin Cancer Res 12, 3184-3192

71 Herbert, B.S. et al. (2005) Lipid modification of GRN163, an N3'-->P5' thiophosphoramidate oligonucleotide, enhances the potency of telomerase inhibition. Oncogene $24,5262-5268$

72 Joseph, I. et al. (2010) The telomerase inhibitor imetelstat depletes cancer stem cells in breast and pancreatic cancer cell lines. Cancer Res 70, 9494-9504

73 Marian, C.O. et al. (2010) The telomerase antagonist, imetelstat, efficiently targets glioblastoma tumor-initiating cells leading to decreased proliferation and tumor growth. Clin Cancer Res 16, 154-163

74 Schepelmann, S. et al. (2007) Suicide gene therapy of human colon carcinoma xenografts using an armed oncolytic adenovirus expressing carboxypeptidase G2. Cancer Res 67, 4949-4955

$75 \mathrm{Yu}$, S.T. et al. (2012) Noninvasive and real-time monitoring of the therapeutic response of tumors in vivo with an optimized hTERT promoter. Cancer 118, 1884-1893 
76 Kojima, T. et al. (2010) In vivo biological purging for lymph node metastasis of human colorectal cancer by telomerase-specific oncolytic virotherapy. Ann Surg 251, 1079-1086

77 Huang, P. et al. (2008) Direct and distant antitumor effects of a telomerase-selective oncolytic adenoviral agent, OBP-301, in a mouse prostate cancer model. Cancer Gene Ther $15,315-322$

78 Nemunaitis, J. et al. (2010) A phase I study of telomerase-specific replication competent oncolytic adenovirus (telomelysin) for various solid tumors. Mol Ther 18, 429-434

79 Fujiwara, T. et al. (2007) Telomerase-specific oncolytic virotherapy for human cancer with the hTERT promoter. Curr Cancer Drug Targets 7, 191-201

80 Brower, V. (2010) Telomerase-based therapies emerging slowly. J Natl Cancer Inst 102, $520-521$

81 Folini, M. et al. (2005) Antisense oligonucleotide-mediated inhibition of hTERT, but not hTERC, induces rapid cell growth decline and apoptosis in the absence of telomere shortening in human prostate cancer cells. Eur J Cancer 41, 624-634

82 Shen, Y. et al. (2008) hTERT-targeted RNA interference inhibits tumorigenicity and motility of HCT116 cells. Cancer Biol Ther 7, 228-236

83 Hemann, M.T. et al. (2001) The shortest telomere, not average telomere length, is critical for cell viability and chromosome stability. Cell 107, 67-77

$84 \mathrm{Fu}, \mathrm{X} . \mathrm{H}$. et al. (2005) Combination of telomerase antisense oligonucleotides simultaneously targeting hTR and hTERT produces synergism of inhibition of telomerase activity and growth in human colon cancer cell line. World J Gastroenterol 11, 785-790

85 Xia, W. et al. (2012) Bioreducible polyethylenimine-delivered siRNA targeting human telomerase reverse transcriptase inhibits HepG2 cell growth in vitro and in vivo. $J$ Control Release 157, 427-436

86 Tamakawa, R.A. et al. (2010) Telomerase inhibition potentiates the effects of genotoxic agents in breast and colorectal cancer cells in a cell cycle-specific manner. Cancer Res 70, 8684-8694

87 Fujiwara, T. et al. (2006) Enhanced antitumor efficacy of telomerase-selective oncolytic adenoviral agent OBP-401 with docetaxel: preclinical evaluation of chemovirotherapy. Int $J$ Cancer $119,432-440$

88 Dong, X. et al. (2009) siRNA inhibition of telomerase enhances the anti-cancer effect of doxorubicin in breast cancer cells. BMC Cancer 9, 133 
89 Goldblatt, E.M. et al. (2009) The telomerase template antagonist GRN163L alters MDAMB-231 breast cancer cell morphology, inhibits growth, and augments the effects of paclitaxel. Mol Cancer Ther 8, 2027-2035

90 Goldblatt, E.M. et al. (2009) Lipid-conjugated telomerase template antagonists sensitize resistant HER2-positive breast cancer cells to trastuzumab. Breast Cancer Res Treat 118, 21-32

91 Mizukoshi, E. et al. (2011) Comparative analysis of various tumor-associated antigenspecific t-cell responses in patients with hepatocellular carcinoma. Hepatology 53, 12061216

92 Agarwal, M. et al. (2008) Inhibition of telomerase activity enhances hyperthermia-mediated radiosensitization. Cancer Res 68, 3370-3378

93 Hacein-Bey-Abina, S. et al. (2003) LMO2-associated clonal T cell proliferation in two patients after gene therapy for SCID-X1. Science 302, 415-419

94 Blasco, M.A. (2007) Telomere length, stem cells and aging. Nat Chem Biol 3, 640-649

95 Buttiglieri, S. et al. (2011) The aging effect of chemotherapy on cultured human mesenchymal stem cells. Exp Hematol 39, 1171-1181 
BOX: Outstanding questions in the development of telomerase based anticancer therapies.

- Do telomerase-based therapies really target the 'Achilles' heel' of cancer? That is, are telomerase functions indispensable for malignant cell survival?

- Are telomerase-based therapies safe in humans, especially with respect to long-term effects of telomerase inhibition/targeting on healthy tissues?

- What are the predictors of tumor response to therapies that target telomerase? In other words, can we select patients with a higher likelihood of responding to this novel anticancer approach?

- Are there resistance mechanisms to telomerase-based therapies? Can these therapies select for tumor clones that downregulate telomerase expression (which would hamper telomerase-

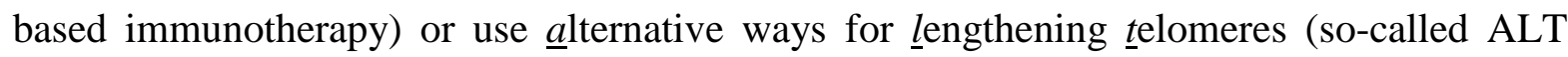
pathways)?

- Is TERC a better target than TERT, or vice versa?

- Are there telomerase inhibitors with better pharmacokinetic properties than currently available compounds?

- Are telomerase-targeting therapies alone sufficient to defeat cancer? Will combinatorial regimens make telomerase-targeting therapies more effective? 
Table 1: Ongoing clinical trials of telomerase-targeting anticancer therapies (www.clinicaltrials.gov)

\begin{tabular}{|c|c|c|c|}
\hline Identifier & Tumor & Design & Intervention \\
\hline NCT01265927 & $\begin{array}{l}\text { Breast cancer } \\
\text { (metastatic) }\end{array}$ & Phase I & $\begin{array}{l}\text { GRN163L }{ }^{\mathrm{a}} \text { (telomerase inhibitor) + trastuzumab (anti- } \\
\text { HER2 monoclonal antibody) }\end{array}$ \\
\hline NCT01342224 & $\begin{array}{l}\text { Pancreatic cancer } \\
\text { (locally advanced) }\end{array}$ & Phase I & GV1001 (TERT-based vaccine) + chemoradiotherapy \\
\hline NCT01568632 & $\begin{array}{l}\text { Refractory solid tumors } \\
\text { and lymphoma }\end{array}$ & Phase I & GRN163L $^{\mathrm{a}}$ (telomerase inhibitor) \\
\hline NCT01579188 & $\begin{array}{l}\text { Non-small cell lung } \\
\text { cancer (inoperable } \\
\text { stage III) }\end{array}$ & Phase III & $\begin{array}{l}\text { GV1001 (TERT-based vaccine) versus placebo (after } \\
\text { curative intent chemoradiotherapy) }\end{array}$ \\
\hline NCT00573495 & $\begin{array}{l}\text { Breast cancer } \\
\text { (advanced) }\end{array}$ & Phase I & TERT and Survivin peptides (vaccine) \\
\hline NCT01242930 & $\begin{array}{l}\text { Multiple myeloma } \\
\text { (previously treated) }\end{array}$ & Phase II & $\begin{array}{l}\text { GRN163L }{ }^{\text {a }} \text { (telomerase inhibitor) as maintenance } \\
\text { therapy }\end{array}$ \\
\hline NCT01256762 & $\begin{array}{l}\text { Breast cancer } \\
\text { (advanced) }\end{array}$ & Phase II $^{b}$ & $\begin{array}{l}\text { GRN163L }{ }^{\mathrm{a}} \text { (telomerase inhibitor) + paclitaxel (with or } \\
\text { without bevacizumab) versus paclitaxel (with or without } \\
\text { bevacizumab) alone }\end{array}$ \\
\hline NCT01137968 & $\begin{array}{l}\text { Non-small cell lung } \\
\text { cancer (previously } \\
\text { treated) }\end{array}$ & Phase II & $\begin{array}{l}\text { GRN163L }{ }^{\mathrm{a}} \text { (telomerase inhibitor) as maintenance } \\
\text { therapy (standard of care versus standard of care + } \\
\text { GRN163L) }\end{array}$ \\
\hline NCT00510133 & $\begin{array}{l}\text { Acute Myelogenous } \\
\text { Leukemia }\end{array}$ & Phase II & GRNVAC1 (TERT-based vaccine) \\
\hline NCT01456065 & $\begin{array}{l}\text { Ovarian cancer } \\
\text { (previously treated) }\end{array}$ & Phase I & $\begin{array}{l}\text { TERT and Survivin peptide loaded dendritic cells } \\
\text { (vaccine) }\end{array}$ \\
\hline NCT00978913 & $\begin{array}{l}\text { Breast cancer or skin } \\
\text { melanoma }\end{array}$ & Phase I & $\begin{array}{l}\text { Dendritic cells transfected with TERT, Survivin and p53 } \\
\text { mRNA (vaccine) }\end{array}$ \\
\hline NCT00425360 & Pancreatic cancer & Phase III & $\begin{array}{l}\text { GV1001 (TERT-based vaccine) + chemotherapy versus } \\
\text { chemotherapy alone }\end{array}$ \\
\hline
\end{tabular}

${ }^{\mathrm{a}}$ GRN163L is also known as imetelstat.

b This trial has been prematurely stopped in September 2012 due to the results of an unplanned interim analysis showing a worse survival outcome in patients receiving GRN163L 


\section{Figure legends}

Figure 1. Telomeres, telomerase, and their relationship to cancer.

The ends of chromosomes are organized in special heterochromatic structures called telomeres, which contain a double-stranded DNA region of TTAGGG repeats and a 150-200 nucleotide-long G-rich single strand. The G-rich overhang invades the doubled-stranded DNA region of the telomere to form a protective telomere T-loop (not shown). The T-loop of the telomere is boud by the shelterin complex, comprising the telomeric repeat binding factor 1 (TRF1), TRF2, repressoractivator protein 1 (RAP1), protection of telomeres protein 1 (POT1), TIN2 organizing protein (TPP1), and TIN2. POT1 binds to the $3^{\prime}$ singled-stranded overhang of the DNA repeats, and TRF1 and TRF2 bind to telomeric double-stranded DNA. TIN2 then binds TRF1 and TRF2 and recruits the TPP1-POT1 complex. Telomerase is a two-component enzyme composed of a catalytic protein subunit (TERT) and the RNA template (TERC); it recognizes the 3 ' hydroxyl group at the end of the G-rich overhang and elongates the telomere. Telomeric DNA uncapping - that is, the loss of normal telomere structure due to either the loss of telomeric repeat sequences or alteration in telomere proteins - can originate from different mechanisms. For example, oncogene-induced replicative stress may include the intrinsic telomere shortening that is associated with cell replication. Dysfunctional telomeres elicit a DNA damage response (DDR) through the activation of upstream kinases, including DNA-dependent protein kinase (DNA-PK) and the ataxiatelangiectasia mutated (ATM) and ataxia-telangiectasia related (ATR) kinases. The DDR can have two opposing outcomes depending on the status of checkpoints usually mediated by tumor suppressor genes (e.g., p53 and p21). p53 activation induces cell cycle arrest, apoptosis, or senescence, negatively affecting stem cell functionality and causing tissue degeneration and, ultimately, organ failure. In p53-deficient cells, the damage proceeds unchecked: the activation of the ATM and ATR kinase pathways leads to mitotic block and cells can then by-pass mitosis and re-enter the S-phase of the cell cycle, becoming polyploid. Polyploidization can lead to genomic instability due to the presence of multiple centrosomes, which will give rise to the random distribution of chromosomes and create aneuploid daughter cells during mitosis. Activation of the non-homologous end joining (NHEJ) pathway leads to end-to-end fusions that initiate cycles of breakage-fusion-bridges. Upon telomere healing, either by telomerase reactivation or by homologous recombination-based mechanisms (e.g., the alternative lengthening of the telomere, ALT), indefinite cell cycling is allowed and stable malignant clones can be generated. 
Figure 2. Schematic view of five telomerase-targeting anticancer strategies.

(a) Given that the telomerase genes TERT and TERC are actively expressed by most malignant cells, vectors designed to encode either oncolytic viruses or suicide genes driven by the TERT or TERC promoters can selectively kill cancer cells. (b) Gene therapy tools such as antisenseoligonucleotides (ASO), small interfering RNAs (siRNAs), or ribozymes can selectively neutralize the mRNA molecules encoding TERT or TERC, ultimately inhibiting telomerase expression. (c) TERT-specific small molecule inhibitors can block telomerase activity. (d) Telomeres, which are physiologically shortened by DNA replication during cell mitosis, can be lengthened by telomerase; however, if a drug stabilizes the G-quadruplex structures that are one of the two modes in which telomeres exist (along with T-loops, see text for more details), telomerase cannot access the G-rich 3'-single strand overhang, which ultimately prevents telomere elongation. (e) TERT protein is degraded by the proteasome, which generates TERT peptides. These peptides are presented on the surface of cells expressing telomerase (such as malignant cells) by class I HLA molecules. If active, specific immunotherapy (i.e. vaccination) regimens elicit an effective immune response to these peptides, cytotoxic T lymphocytes can recognize and kill malignant cells. 


\section{GLOSSARY}

ASO (anti-sense oligonucleotide): a class of short strand deoxyribonucleotide analogues that sequence-specifically hybridize with complementary mRNA via Watson-Crick base pairing. Formation of the ASO-mRNA heteroduplex either triggers the activity of RNaseH (leading to mRNA degradation) or induces translational arrest by steric hindrance of ribosomal activity, ultimately downtregulating target protein expression and activity.

BIBR1532: Non-competitive small molecule inhibitor of both TERT and TERC.

BRACO19: a G-quadruplex stabilizer that potently inhibits telomerase.

Cancer stem cell: An immortal cancer cell with self-renewing capacity and the ability to indefinitely sustain a tumor cell population.

Cancer vaccine: A therapeutic preparation containing tumor-associated antigens (TAAs) - or peptides derived from these proteins - that elicit an adaptive immune response against malignant cells expressing those antigens. In dendritic cell-based vaccines, autologous dendritic cells are first loaded ex vivo with peptides or transfected with genes encoding tumor associated antigens and then injected into patients.

Cell senescence: Cell cycle arrest following a limited number of cell divisions, typically triggered by one or more dysfunctional telomeres.

Dendritic cells: Professional antigen presenting cells (APCs) that present protein-derived peptides loaded on class I and class II HLA molecules to CD8+ (cytotoxic) and CD4+ (helper) Tlymphocytes, respectively.

G-quadruplex: a four-stranded nucleic acid structure that consists of a square arrangement of guanines stabilized by Hoogsteen hydrogen bonds. G-quadruplex structures form within or between guanine-rich strands of DNA, including telomeres.

GRNVAC1: Anticancer vaccine made of mature autologous (i.e., obtained from the patient) dendritic cells transfected with mRNA encoding a TERT-LAMP (lysosomal associated membrane protein) fusion construct.

GV1001: Telomerase-based cancer vaccine made of a 16-mer TERT peptide.

Imetelstat: an antisense oligonucleotide (also known as GRN163L) complementary to the TERC mRNA that inhibits telomerase expression.

IRES (internal ribosomal entry site): a sequence within an mRNA that allows translation initiation independent of a 5'-terminal mRNA cap.

Ribozyme: an RNA molecule with a well-defined tertiary structure that enables it to perform a chemical reaction; a catalytic RNA molecule. 
siRNA (small interfering RNA): a class of double-stranded RNA 20-25 nucleic acids long that mediate RNA interference, that is, inhibition of the expression of specific genes or mRNAs by a complementary nucleotide sequence.

Suicide gene: A gene that, when expressed, leads to death of the hosting cell. Expression of suicide genes is controlled by promoters that are preferentially activated in target cells. For example, TERC or TERT promoters are used within tumors. Examples of suicide genes are those that encode proteins that control the replication of oncolytic viruses or enzymes that convert a pro-drug into a toxic compound.

Telomelysin: Attenuated adenovirus 5 vector, also known as OBP-301, in which the TERT promoter element drives expression of the E1A and E1B genes linked with an internal ribosome entry site (IRES).

Telomerase: A ribonucleoprotein made of a protein (TERT) and an RNA component (TERC). Its best characterized activity is to elongate telomeres, but is also appears to have extra-telomeric functions.

Telomere: The ends of linear chromosomes are made of repetitive TTAGGG DNA sequences associated with telomere binding proteins; this structure protects chromosome ends from being recognized as DNA double stand breaks by the DNA repair machinery.

Telomere uncapping: Loss of proper telomere structure due to either severe loss of telomeric repeat sequences (telomere shortening) or alteration in telomeric proteins. In both cases a cascade of events called the DNA damage response (DDR) is elicited.

Telomestatin: a small molecule G-quadruplex stabilizer that acts as a potent telomerase inhibitor.

TERC: telomerase RNA component, the RNA template of telomerase.

TERT: telomerase reverse transcriptase, the catalytic subunit of telomerase.

Vx-001: Telomerase-based cancer vaccine made of a 9-mer TERT peptide modified at position 572 to increase its immunogenicity. 